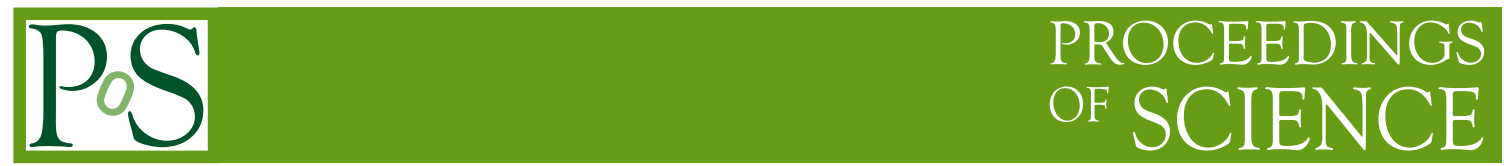

\title{
Gluon saturation and gluon density
}

\section{Krzysztof Kutak*}

The H. Niewodniczański Institute of Nuclear Physics PAN, Radzikowskiego 152, Kraków, Poland E-mail: krzysztof.kutak@ifj.edu.pl

I overview an approach to search for gluon saturation focused on studies of jets. First of all I will introduce factorization and gluon density. In the next step the formalism is applied to study saturation effects in the production of forward dijets in proton-lead collisions at the Large Hadron Collider. Such configurations, with both jets produced in the forward direction, probe the gluon density of the lead nucleus at small longitudinal momentum fraction.

The 33rd International Symposium on Lattice Field Theory

14 -18 July 2015

Kobe International Conference Center, Kobe, Japan*

${ }^{*}$ Speaker. 


\section{Introduction}

The production of hadronic final states at the Large Hadron Collider (LHC) offers unprecedented opportunities to test parton densities in various kinematic regions. Of particular interest is the forward region, where it is possible to construct hadronic observables that allow to probe parton densities of one of the colliding hadrons at longitudinal fractions $x \sim 10^{-5}$. At such low values of $x$, on theoretical grounds, one expects "low- $x$ " effects to be relevant, in particular the phenomenon of gluon saturation [1].

In QCD, saturation is described by non-linear evolution equations for the gluon density, which resum a subset of diagrams generating contributions of the form $\alpha_{s} \ln 1 / x$. The solutions of small- $x$ evolution equations, together with suitable initial conditions, provide parton densities, which then need to be convoluted with appropriate hard matrix elements in order to obtain predictions for measurable cross sections. The Color Glass Condensate (CGC) (see [2] and references therein) and High Energy Factorization (HEF) [3] are two QCD-based frameworks which can be used for phenomenological studies.

The CGC approach has been very successful in describing forward di-hadron production at RHIC $[4,5,6]$, in particular it predicted the suppression of azimuthal correlations in d+Au collisions compared to $\mathrm{p}+\mathrm{p}$ collisions [7], which was observed later experimentally. It has now become necessary to extend the validity of the CGC predictions from RHIC kinematics to the LHC. There, the relevant observables involve high- $p_{t}$ jets, as opposed to individual hadrons with $p_{t}$ of the order of a few $\mathrm{GeV}$ at RHIC. Furthermore the advantage of jet observables as compared to hadrons is that they are less sensitive to large uncertainties from fragmentation functions. In the context of the LHC, as a dedicated process to look for saturation we will consider forward dijet production in $\mathrm{p}+\mathrm{Pb}$ and $\mathrm{p}+\mathrm{p}$ collisions. Comparisons of $\mathrm{p}+\mathrm{Pb}$ and $\mathrm{p}+\mathrm{p}$ cross sections for the same observables can provide some evidences for parton saturation since such effects are further enhanced by increasing the atomic number of one of the colliding particles. In this contribution study we overview results obtained using HEF the more complete study which uses unified framework of CGC and HEF [20] is on the way.

\section{High Energy Factorization}

In order to describe the collisions of hadrons at high energies in the framework of perturbative quantum chromodynamics (pQCD) one uses factorization, which allows to decompose the cross section for the process into parton distribution functions (gluon and quarks) and so-called matrix elements defining the production of final states at a given hard scale. In the High Energy Factorization formalism $[1,3]$ the initial state off shell gluons (dominating partons in the considered kinematics) have virtuality $k_{t}^{2}$ and carry a small fraction $x P$ of longitudinal proton's momentum $P$. The factorization formula assumes the form:

$$
\begin{gathered}
\frac{d \sigma}{d y_{1} d y_{2} d^{2} p_{1 t} d^{2} p_{2 t}}=\sum_{c, d} \int \frac{d^{2} k_{1 t}}{\pi} \frac{d^{2} k_{2 t}}{\pi} \frac{1}{16 \pi^{2}\left(x_{1} x_{2} S\right)^{2}}{\overline{\mid \mathscr{M}_{g^{*} g^{*} \rightarrow c d}}}^{2} \delta^{2}\left(k_{1 t}+k_{2 t}-p_{1 t}-p_{2 t}\right) \\
\times \mathscr{F}_{A}\left(x_{1}, k_{1 t}^{2}\right) \mathscr{F}_{B}\left(x_{2}, k_{2 t}^{2}\right) \frac{1}{1+\delta_{c d}},
\end{gathered}
$$


where $\mathscr{F}_{i}$ is the unintegrated gluon distribution in the hadron, while $\mathscr{M}_{g^{*}} g^{*} \rightarrow c d$ is a matrix element, which is a factor that determines the probability of a particular process. The transversal momenta of final state particles are given by $p_{1 t}, p_{2 t}$. Gluon distributions are objects satisfying evolution equations derived within QCD summing dominant diagrams in the given range of kinematic variables. Summing diagrams under the assumption of some initial functional form of nonperturbative gluon distribution leads to the perturbative evolution of the nonperturbative distribution. In the limit when the dominant scale in the considered kinematics is given by the energy of the collision, and parton densities are not probed at too low values of $x$ one derives an equation known as the BFKL (Balitsky, Fadin, Kuraev, Lipatov) equation [13]. However, it turns out that the solution of the BFKL equation just like standard renormalization group based DGLAP (Gokshitzer, Gribov, Altarelli, Parisi) equations leads to a power-like growth of the density of gluons with decreasing values of the parameter $x$. This is in conflict with the unitarity bound [1]. Taking into account unitarization corrections, which introduce the recombination of gluons, leads to nonlinear evolution equations $[8,9,10]$ that allow for saturation. Saturation is associated with dominance of the gluon density by the effects of gluon recombination, see Fig. 1 (right), which leads to the generation of a new semi-hard scale in the system of gluons called saturation scale $Q_{s}$. The effect of saturation is relevant especially for small values of $x$ and $k_{t}^{2}$. The existence of such a scale on the level of theoretical calculations changes the growth of the proton structure function $F_{2}\left(x, Q^{2}\right)$ as a function of $x$ from power like to logarithmic. Gluon saturation effects were investigated for the first time in deep inelastic scattering (DIS) of electron on proton, and as mentioned in the introduction there are hints that saturation occurs in nature. Later, the search for saturation was carried out in hadron-hadron collisions. In the LHC era, it is necessary to use a formalism which takes into account the effects of resumed corrections of higher orders in perturbation theory. This is necessary in order to be able to go from RHIC to the LHC kinematics, and thus to be able to describe the final states produced in a wide range of momentum and pseudorapidity accessible at the LHC.

\section{Non-linear evolution of the unintegrated gluon distributions}

The formal definition of unintegrated gluon distribution assumes the form

$$
\mathscr{F}\left(x, k_{t}\right)=2 \int \frac{d \xi^{+} d^{2} \xi}{(2 \pi)^{3} p_{A}^{-}} e^{i x p_{A}^{-} \xi^{+}-i k_{t} \cdot \xi}\left\langle A\left|\operatorname{Tr}\left[F^{i-}\left(\xi^{+}, \xi\right) F^{i-}(0)\right]\right| A\right\rangle,
$$

where $F^{i-}$ are components of the gluon field strength tensor, must be also supplemented with gauge links, in order to render such a bi-local product of field operators gauge invariant. The gauge links are path-ordered exponentials, with the integration path being fixed by the hard part of the process under consideration. Therefore, unintegrated gluon distributions are process-dependent. One can show that the formal definition is satisfied by the BK equation in momentum space, for 
the unintegrated gluon density $\mathscr{F}$, reads [11]:

$$
\begin{aligned}
\mathscr{F}_{p}\left(x, k^{2}\right)= & \mathscr{F}_{p}^{(0)}\left(x, k^{2}\right) \\
+ & \frac{\alpha_{s} N_{c}}{\pi} \int_{x}^{1} \frac{d z}{z} \int_{k_{0}^{2}}^{\infty} \frac{d l^{2}}{l^{2}}\left\{\frac{l^{2} \mathscr{F}_{p}\left(\frac{x}{z}, l^{2}\right)-k^{2} \mathscr{F}_{p}\left(\frac{x}{z}, k^{2}\right)}{\left|l^{2}-k^{2}\right|}+\frac{k^{2} \mathscr{F}_{p}\left(\frac{x}{z}, k^{2}\right)}{\left|4 l^{4}+k^{4}\right|^{\frac{1}{2}}}\right\} \\
& \quad-\frac{2 \alpha_{s}^{2}}{R^{2}}\left[\left(\int_{k^{2}}^{\infty} \frac{d l^{2}}{l^{2}} \mathscr{F}_{p}\left(x, l^{2}\right)\right)^{2}+\mathscr{F}_{p}\left(x, k^{2}\right) \int_{k^{2}}^{\infty} \frac{d l^{2}}{l^{2}} \ln \left(\frac{l^{2}}{k^{2}}\right) \mathscr{F}_{p}\left(x, l^{2}\right)\right] .
\end{aligned}
$$

where $R$ is a radius of the target proton In this formulation one can relatively easily include dominant corrections of higher orders as it has been done in [11, 12]. In principle, the gluon density in the HEF framework $\mathscr{F}_{p}$ evolves with $x$ according to the BFKL evolution. However, BK evolution introduced above can also be used to estimate effects of non-linear corrections to the BFKL evolution.

\section{In search for saturation: forward-forward dijet production at the LHC}

In $\mathrm{p}+\mathrm{A}($ or $\mathrm{p}+\mathrm{p})$ collisions, particle production at forward rapidities is sensitive to large- $x$ partons from the proton, while the target nucleus (or the other proton) is probed deep in the small- $x$ regime. To compute cross sections in such an asymmetric situation, the appropriate formulation is the so-called hybrid factorization [16].

In the hybrid formalism, the large- $x$ partons are described in terms of the usual parton distribution functions of collinear factorization $f_{i / p}$, with a scale dependence given by DGLAP evolution equations, while the small- $x$ gluons of the nucleus are described by a transverse momentum dependent distributions, which evolve towards small $x$ according to non-linear equations.

The double-inclusive particle production in the hybrid HEF reads:

$$
\frac{d \sigma^{p A \rightarrow \text { dijets }+X}}{d y_{1} d y_{2} d^{2} p_{1 t} d^{2} p_{2 t}}=\sum_{a, c, d} \frac{1}{16 \pi^{3}\left(x_{1} x_{2} S\right)^{2}}\left|\overline{\mathscr{M}}_{a g^{*} \rightarrow c d}\right|^{2} x_{1} f_{a / p}\left(x_{1}, \mu^{2}\right) \mathscr{F}_{A}\left(x_{2},\left|p_{1 t}+p_{2 t}\right|\right) \frac{1}{1+\delta_{c d}} .
$$

The quantities $\left|\overline{\mathscr{M}_{a g^{*} \rightarrow c d}}\right|^{2}$ are $2 \rightarrow 2$ polarization-averaged matrix elements with one off-shell incoming small- $x$ gluon and the other partons on-shell. The following partonic sub-processes contribute to the production of the dijet system:

$$
\begin{gathered}
q g \rightarrow q g, \quad g g \rightarrow q \bar{q}, \quad g g \rightarrow g g . \\
x_{1}=\frac{1}{\sqrt{S}}\left(p_{1 t} e^{y_{1}}+p_{2 t} e^{y_{2}}\right), \quad x_{2}=\frac{1}{\sqrt{S}}\left(p_{1 t} e^{-y_{1}}+p_{2 t} e^{-y_{2}}\right),
\end{gathered}
$$

and $\sqrt{S}$ is the center of mass energy of the p+A system. The corresponding amplitudes were computed in [17]. We move now to the numerical results for forward dijet production at the LHC in $\mathrm{p}+\mathrm{p}$ and $\mathrm{p}+\mathrm{Pb}$ collisions.

Our predictions were generated with the forward region defined as the rapidity range $3.2<$ $y<4$.9. The two hardest jets (sorted according to their $p_{t}, p_{t 1}>p_{t 2}$ ) are required to lie within this region. It turns out that the corrections of higher orders to the BK equation, are quite large therefore in order to provide realistic predictions we performed our computations using extension 

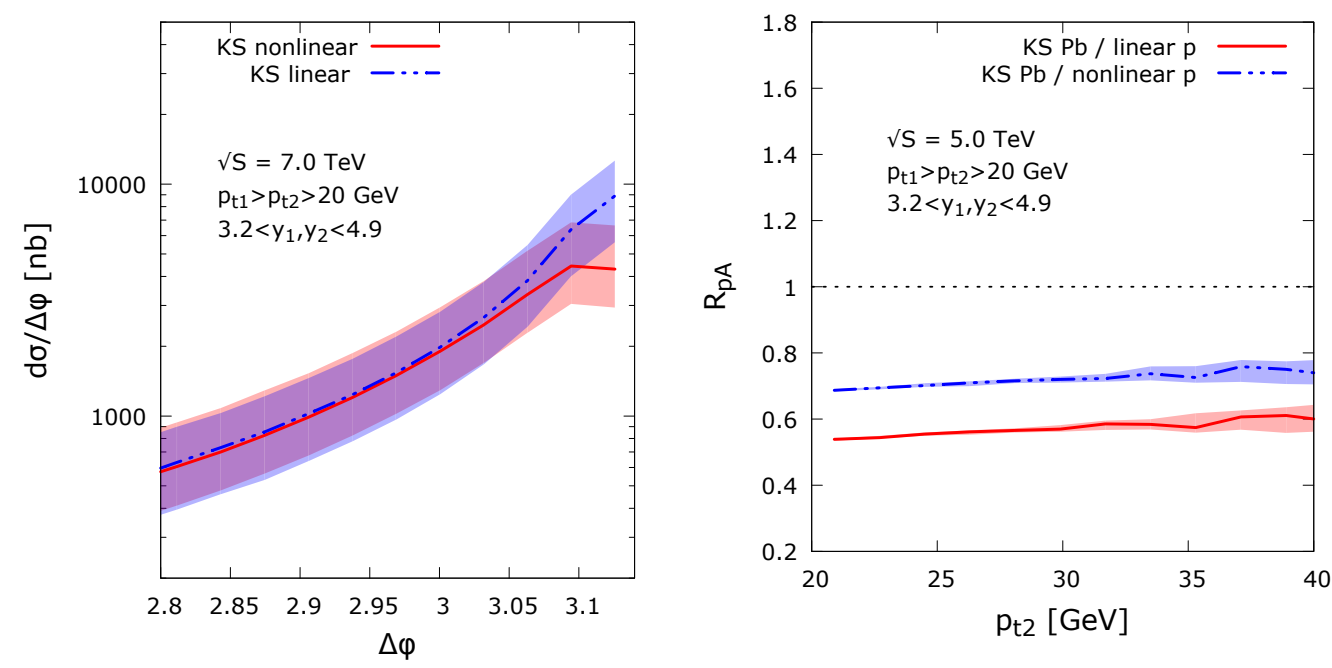

Figure 1: Comparisons of predictions obtained with a linear vs non-linear proton evolution, for the differential cross section in $p+p$ collisions as a function of the azimuthal angle (left), and the nuclear modification factor as a function of $p_{t}$ of the subleading jet. KS gluon densities are used, with parameter $c=1$ in the nuclear case.

of BK evolution equation which solution and fit to HERA data results in KS gluon density [18]. For the collinear PDFs, which also enter the HEF formula (4.1), we took the general-purpose CT10 set. For the central value of the factorization and renormalization scale, we chose the average transverse momentum of the two leading jets, $\mu_{F}=\mu_{R}=\frac{1}{2}\left(p_{t 1}+p_{t 2}\right)$.

We illustrate in Fig. 1 the impact of saturation effects in the KS evolution by switching off the non-linear term in the evolution. We note that the parameters of this alternative gluon distribution for the proton, obtained with linear evolution, are re-adjusted in order to keep a good description of DIS data from HERA. The left plot shows the impact of non-linear effects on the differential cross section in $\mathrm{p}+\mathrm{p}$ collisions as a function of the azimuthal angle, and it is large, as expected, near $\Delta \Phi=\pi$. The right plot shows, in the case of the sub-leading jet $p_{t}$ dependence, by how much $R_{p P b}$ is reduced if the nuclear gluon density of lead is still subject to non-linear effects, but not proton one. Of course with the KS gluon distributions, by construction, if non-linear evolution is switched off both in the proton and nuclear cases, then $R_{p P b}=1$.

\section{Summary}

In this paper we review on search for gluon saturation with studies of forward-forward dijet production and we argued that this process is particularly attractive from low- $x$ point of view. Using the High Energy Factorization approach (Eq. (4.1)). As the results to emphasize the role of nonlinearity I just flashed just one distribution and plot nuclear modification factors in $\mathrm{p}+\mathrm{Pb} v \mathrm{p}+\mathrm{p}$ collisions,. The complete study has been presented in [19] and the theoretical extension in order to account for complete CGC effects in [20]. 


\section{Acknowledgements}

This research has been supported by Narodowe Centrum Nauki with Sonata Bis grant DEC2013/10/E/ST2/00656. The results presented in this contribution were obtained in common paper with Piotr Kotko, Cyrille Marquet, Sebastian Sapeta and Andreas van Hameren whom I acknowledge for fruitful collaboration.

\section{References}

[1] L. V. Gribov, E. M. Levin and M. G. Ryskin, Phys. Rept. 100 (1983) 1.

[2] F. Gelis, E. Iancu, J. Jalilian-Marian and R. Venugopalan, Ann. Rev. Nucl. Part. Sci. 60 (2010) 463.

[3] S. Catani, M. Ciafaloni and F. Hautmann, Nucl. Phys. B 366 (1991) 135.

[4] J. L. Albacete and C. Marquet, Phys. Rev. Lett. 105 (2010) 162301.

[5] A. Stasto, B. -W. Xiao and F. Yuan, Phys. Lett. B 716 (2012) 430.

[6] T. Lappi and H. Mantysaari, Nucl. Phys. A 908 (2013) 51.

[7] C. Marquet, Nucl. Phys. A 796 (2007) 41.

[8] I. Balitsky, Nucl. Phys. B463 (1996) 99-160.

[9] Y. V. Kovchegov, Phys. Rev. D 60 (1999) 034008.

[10] Y. V. Kovchegov, Phys. Rev. D 61 (2000) 074018.

[11] K. Kutak and J. Kwiecinski, Eur. Phys. J. C 29 (2003) 521 [hep-ph/0303209].

[12] K. Kutak and A. M. Stasto, Eur. Phys. J. C 41 (2005) 343 [hep-ph/0408117].

[13] L. N. Lipatov, Sov. J. Nucl. Phys. 23, 338 (1976);

E. A. Kuraev, L. N. Lipatov and V. S. Fadin, Sov. Phys. JETP 45, 199 (1977);

I. I. Balitsky and L. N. Lipatov, Sov. J. Nucl. Phys. 28, 822 (1978).

[14] A. M. Stasto, B. -W. Xiao and D. Zaslavsky, Phys. Rev. Lett. 112 (2014) 012302.

[15] Z. -B. Kang, I. Vitev and H. Xing, arXiv:1403.5221 [hep-ph].

[16] A. Dumitru, A. Hayashigaki and J. Jalilian-Marian, Nucl. Phys. A 765 (2006) 464.

[17] M. Deak, F. Hautmann, H. Jung and K. Kutak, "Forward Jet Production at the Large Hadron Collider," JHEP 0909 (2009) 121.

[18] K. Kutak and S. Sapeta, Phys. Rev. D 86 (2012) 094043 [arXiv:1205.5035 [hep-ph]].

[19] A. van Hameren, P. Kotko, K. Kutak, C. Marquet and S. Sapeta, Phys. Rev. D 89 (2014) 9, 094014 [arXiv:1402.5065 [hep-ph]].

[20] P. Kotko, K. Kutak, C. Marquet, E. Petreska, S. Sapeta and A. van Hameren, JHEP 1509 (2015) 106 [arXiv:1503.03421 [hep-ph]]. 